\title{
eJRIEPS
}

Ejournal de la recherche sur l'intervention en éducation physique et sport

$9 \mid 2006$

Varia

Intervention et appropriation de « savoirs » sur les techniques sportives. Une innovation en formation universitaire des étudiants de licence STAPS

Richard Refuggi, Eric Louis, Attiklémé Kossivi et Michel Deridder

\section{(C) OpenEdition}

Édition électronique

URL : https://journals.openedition.org/ejrieps/7468

DOI : $10.4000 /$ ejrieps.7468

ISSN : 2105-0821

Éditeur

ELLIADD

Édition imprimée

Pagination : 67-81

\section{Référence électronique}

Richard Refuggi, Eric Louis, Attiklémé Kossivi et Michel Deridder, « Intervention et appropriation de « savoirs » sur les techniques sportives. Une innovation en formation universitaire des étudiants de licence STAPS », eJRIEPS [En ligne], 9 | 2006, mis en ligne le 01 janvier 2006, consulté le 29 octobre 2021. URL : http://journals.openedition.org/ejrieps/7468; DOI : https://doi.org/10.4000/ejrieps.7468

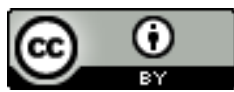

La revue eJRIEPS est mise à disposition selon les termes de la Creative Commons Attribution 4.0 International License. 
Intervention et appropriation de « savoirs " sur les techniques sportives. Une innovation en formation universitaire des étudiants de licence STAPS.

R. Refuggi*, Eric Louis, Attiklémé Kossivi ${ }^{\star \star}$, Michel Deridder UFR APS Université joseph Fourier, Grenoble. *LEMME Toulouse. ${ }^{* *}$ INJEPS Porto-Novo Benin

Résumé

Consécutivement à la déclaration de Bologne (1999), les universités françaises ont eu à réorganiser leurs formations pour satisfaire aux nouvelles normes européennes (Camy, 2005). Ce fut l'occasion pour les Unités de Formation et de Recherche en Activité Physique et Sportive (UFR.APS) de profiter de la mise en place d'un nouveau format de formation (Licence- Master et Doctorat) pour repenser les contenus des enseignements à y dispenser (Terral, 2005). A Grenoble, ceux-ci ont été reconsidérés au niveau du premier cycle de la formation (licence) et cela dans le but de mieux les adapter aux problématiques de l'intervention dans le domaine du sport et de l'éducation physique. C'est ainsi que trois spécialités ont été habilitées par le ministère s'inscrivant toutes dans une même mention intitulée : Sciences et techniques des activités physiques et sportive (STAPS) et intervention.

La référence aux pratiques et problématiques de l'intervention pour définir les contenus à enseigner dans ce domaine de formation apparaît nécessaire non seulement parce que la volonté d'enseigner, entraîner, rééduquer... correspond au projet professionnel de l'immense majorité des étudiants qui y sont engagés. Mais aussi et surtout parce qu'elle paraît être un bon moyen pour rompre avec la logique académique qui préside aujourd'hui à la conception des programmes des formations générales (non professionnelles) proposées au sein des universités et ce aussi bien en France qu'aux Etats Unis (Siedentop, 2002). Une logique qui, si on n'y prend garde, peut conduire selon nous à un éclatement de la formation (générale) initiale en « un agrégat de discours (savants) divers, parallèles et isolés, plus ou moins lâchement indexés à ces pratiques » (Durand, 2001, 13). Cela peut rendre celle-ci guère signifiante et performante au regard des compétences exigibles à l'exercice d'une des professions relevant de ce secteur d'activités (Siedentop,2002). 
Chacune de ces spécialités laisse place à une diversité de parcours que les étudiants peuvent choisir en fonction de ce qu'ils entendent faire ultérieurement de leur diplôme et de leurs profils de compétence. Ces parcours reposent sur un tronc commun de formation assez important qui est lui-même structuré en quatre dimensions : scientifique, technologique, sportive, personnelle et pré-professionnelle. Les trois premières dimensions regroupent un ensemble d'unités d'enseignement (UE) spécifiques à ce domaine de formation que sont les STAPS dont la liste est présentée en annexe.

Les enseignements constitutifs de chacune de ces dimensions ont été élaborés à partir d'une démarche relevant de l'ingénierie (Legendre, 1993 ; Ardouin, 2003) qui implique une analyse du problème que la formation entendait résoudre, la conception d'un nouveau dispositif, sa mise en œuvre et le suivi de celle-ci ainsi que son évaluation. Une démarche conduite d'une manière plus concourante que linéaire (Leboterf, 2004) au sens où le projet de départ, qui n'était structuré qu'autour de quelques idées ou velléités générales, a dû être précisé voire rectifié chemin faisant. Cette élaboration est encore inachevée au moment où cet article est écrit. Elle donne lieu aujourd'hui encore à de multiples négociations et régulations pouvant générer quelques conflits entre acteurs. En conséquence, son avancement se fait par accords successifs autour d'ébauches, de maquettes, ou d'esquisses, bref autour « d'objets intermédiaires » (Le Boterf, 2004), dont fait partie cet article.

La mise en œuvre de cette démarche a nécessité la création de nouvelles instances de travail. C'est ainsi qu'un groupe de pilotage a été institué avec des enseignants volontaires et reconnus par leurs pairs. Celui-ci est constitué de huit personnes. II a en charge la conception, l'organisation de la réflexion collective et son animation afin d'élaborer les éléments du programme en temps limité, mais aussi la régulation du projet de formation. Les membres de ce groupe ont à animer également une réflexion avec les enseignants concernés afin d'arrêter les principales orientations et dispositions pratiques au sein de trois groupes de réflexion et de propositions, un par dimension de la formation spécifique aux STAPS. Orientations et dispositions que les responsables des différentes unités d'enseignement programmées ont in fine à mettre en œuvre avec les enseignants qui y interviennent.

C'est à la présentation de ce projet que nous allons nous intéresser dans le cadre de cet article, en réduisant notre propos à la partie concernant la dimension technologique de la formation. Ce processus étant encore en cours de réalisation, nous nous attacherons à présenter ce qui relève plus particulièrement de la phase d'investigation par rapport celle relative à la mise en œuvre du projet (Ardouin, 2003). C'est ainsi que nous présenterons 
dans un premier temps le problème que l'on entendait résoudre par cette formation, puis, dans un deuxième temps, les éléments essentiels de ce projet de formation et préciserons enfin les idées à partir desquelles la formation était jusqu'alors pensée et avec lesquelles ce projet tend à rompre.

\section{Le problème que ce projet de formation entend résoudre.}

Au départ la difficulté consiste à mobiliser dans le cadre de l'intervention des connaissances sur les APS dès lors que celles-ci se démarquent des aspects gestuels les plus immédiatement observables. Cette difficulté a été en effet repérée de manière récurrente aussi bien chez les étudiants à l'issue de leur formation en STAPS que chez les professionnels en exercice.

1. 1. Au niveau de la formation initiale :

La difficulté que nous venons d'énoncer est régulièrement notée dans le cadre des réunions des jurys de diplômes ou encore lors des rapports de jury des concours notamment et ce malgré les efforts consentis dans les formations pour satisfaire à ces exigences. Nous l'avons nous-mêmes constatée lors d'un enseignement de maîtrise STAPS au cours duquel les étudiants avaient à enseigner une activité physique à leurs pairs puis à analyser le contenu de leur intervention: lorsqu'ils avaient à prendre des décisions quant au contenu à enseigner à leurs camarades, les étudiants n'utilisaient que très rarement les connaissances qui leurs avaient été dispensées antérieurement. De ce fait, les décisions prises ne se démarquaient pas ou très peu de celles d'une personne n'ayant pas reçue de formation. Ce constat était d'autant plus surprenant qu'il contrastait avec le niveau de discours que ces étudiants étaient capables de produire sur l'enseignement. En fait, ce «bien parlé », le plus souvent convenu, que leur formation antérieure avait grandement contribué à former ${ }^{1}$, était en fait peu ou pas du tout opératoire. La fonctionnalité didactique de ces connaissances, c'est à dire leur utilité pour agir et analyser la prestation des pratiquants dont ils avaient la charge ne semblait pas faire partie de leur monde. A contrario, l'intérêt porté à cet enseignement et les perspectives qu'il permettait d'entrevoir, mais aussi l'absence de temps dont nous disposions pour traiter les thèmes abordés, ont amené ces étudiants à nous reprocher le

\footnotetext{
1 Nous pensons que ce phénomène est accentué lorsque la formation dispensée dans le cadre de la licence tend à être assimilée par les étudiants à une préparation aux différentes épreuves du concours de recrutement (CAPEPS).
} 
fait de ne pas l'avoir programmé plus tôt dans leur cursus et sur un spectre plus large d'activités sportives.

Cette difficulté s'accompagne le plus souvent d'un rejet des connaissances scientifiques et technologiques dispensées initialement au nom de leur (apparente) «non utilité ». Cette position est semble-t-il renforcée par une mise en situation d'enseignement trop précoce lors des stages en établissement en licence et ce, sans que l'on puisse en contrôler les conditions (type d'établissement, compétences et rôle des conseillers pédagogiques...). Une telle mise en situation, bien qu'essentielle dans une perspective de préprofessionnalisation (c'est à dire de la découverte d'un métier), apparaît en effet éminemment problématique au regard du développement de certaines compétences professionnelles. En effet, les constats effectués par Durand (1996) à propos de la formation des futurs enseignants, sont aussi d'actualité en ce qui concerne la formation d'intervenants en sport (débouchés potentiels des filières STAPS). Cet auteur souligne notamment [qu'] « actuellement la formation consiste souvent à placer les enseignants débutants, de façon accompagnée ou non, face à une tâche d'un niveau de complexité maximale (objectifs pédagogiques élevés et nombreux, contraintes liées au contexte non aménagées). Cette situation est de nature à induire chez eux des stratégies de réduction de complexité, d'effacement ou de contournement du problème, plutôt que des démarches d'acquisition délibérée de compétences préalablement repérées. II serait utile de concevoir des plans de formation jalonnés par des mises en situation d'enseignement conçues en fonction d'objectifs de développement des compétences professionnelles partielles. » (Durand, 1996, p. 83). Elle est aussi contre productive par rapport à la nécessité d'avoir à apprendre autres choses que les techniques et les règlements pour enseigner les activités physiques sportives et artistiques (APSA).

1. 2. Au niveau des professionnels en exercice :

La relecture des certaines recherches, conduites notamment en didactique de l'éducation physique et sportive (EPS), conduit à penser que cette difficulté existe également chez les enseignants en exercice et même si ceux-ci sont chevronnés. C'est en effet ce qui ressort des recherches relevant d'une démarche d'ingénierie didactique, menées principalement dans le cadre de l'Institut National de la Recherche Pédagogique (INRP). Ces recherches ont été engagées dans le but d'élaborer un enseignement qui soit susceptible de faire évoluer significativement la motricité usuelle des élèves et ce en concevant des contenus non plus à partir d'une centration sur les traits les plus immédiatement observables des gestes réalisés mais à partir d'une centration sur les transformations du sujet, nécessaires à leur apparition (Amade- Escot et Marsenach, 1995). Selon ces auteurs, cette nouvelle 
manière de concevoir les contenus a nécessité l'élaboration d'un cadre interprétatif de l'activité adaptative des élèves à partir des connaissances scientifiques disponibles et des connaissances d'experts des activités sportives concernées.

Les recherches menées ultérieurement sur la diffusion des produits de ces ingénieries (contenus et dispositifs d'enseignement) ont montré que celle-ci était problématique. Une première étude (Amade- Escot et Marsenach, 1995), réalisée auprès d'enseignants volontaires et ayant suivi une formation préalable, a consisté à comparer leurs projets d'enseignement avec celui de l'ingénierie. II ressort de cette comparaison une difficulté persistante à fonctionner sur cette nouvelle conception des contenus. Les enseignants en exercice semblent avoir eu des difficultés à assimiler le cadre interprétatif de l'activité adaptative des élèves ayant permis l'élaboration des contenus proposés dans le cadre de l'ingénierie. Une seconde étude a été réalisée à partir d'une observation de ce qui se passait en cours (Amade Escot et Léziart, 1996). En étudiant plus particulièrement les interactions didactiques observées lors de séances conduites par certains de ces enseignants, ces recherches ont mis en évidence l'ingéniosité de ces derniers pour maintenir coûte que coûte une relation didactique avec leurs élèves et en même temps le fait que ce maintien pouvait s'accompagner d'une perte de sens ou d'une transformation du savoir en jeu, voire de son évanouissement. Ceci amène à poser la question de la diffusion des produits d'ingénierie non plus en terme d'application mais en terme d'articulation entre deux activités créatrices de nature différentes : l'ingénierie et le génie didactique (Mercier, Lemoyne, Rouchier, 2001). II faut également situer l'origine des difficultés rencontrées par les enseignants dans leur « épistémologie » c'est à dire dans la théorie de la connaissance enseignée, qui est en jeu dans leur pratique et qui, en arrière plan et de manière le plus souvent tacite, surdétermine leur ingéniosité pratique.

Cette explication invite à interroger le rapport entre l'épistémologie des professeurs et la formation qu'ils ont reçue. Le travail réalisé par Attiklélé (2002) sur les programmes de natation en EPS s'inscrit dans cette perspective. Après avoir constaté que les enseignants des établissements étudiés avaient du mal à s'entendre sur les éléments techniques qu'ils pouvaient faire acquérir à leurs élèves, ce chercheur a établi que l'origine de cette difficulté pouvait résider dans le fait que les savoirs auxquels il est possible de se référer pour débattre de manière éclairée sur ce sujet et essayer de faire entendre raison à ses pairs ne faisaient pas partie de leurs univers cognitifs. Pour certains, ces savoirs ne devaient même pas en faire partie. En effet, après avoir refusé d'entrer dans ce genre de réflexion, ceux-ci ont considéré qu'elle ne relevait pas de leur compétence mais de celle de spécialistes de l'activité physique enseignée. Ce chercheur a alors investigué la 
formation qu'ils avaient reçues en natation à partir du souvenir qu'ils en avaient. De cette investigation, il ressort que les enseignants interrogés n'ont jamais été confrontés à ce genre d'exercice dans le cadre de leur propre formation et qu'ils n'ont par conséquent jamais eu à manipuler ce type de savoir. Ceci l'amène à considérer que l'attitude de ces enseignants, qui considéraient le fait de définir les éléments techniques à faire acquérir à leurs élèves comme ne relevant pas de leur champ de compétence. En fait, ils réfléchissaient à une des options fondamentales sur laquelle était et est toujours fondée le fonctionnement de la formation dispensée dans les UFRAPS que l'on a l'habitude d'appeler la polyvalence en APS (c'est-à-dire la formation à propos des diverses activités physiques et sportives). Poids de la formation initiale chez les enseignants d'EPS que d'autres auteurs comme Collinet (2005) ont également mis en évidence.

Cette difficulté repérée aussi bien chez les étudiants que chez les enseignants en exercice pose, selon nous, la question de la nature des connaissances dispensées sur les techniques sportives ou artistiques et de leur articulation avec les problématiques de l'intervention dans les APS. Une question qui dépasse la seule formation des enseignants d'EPS, et peut être posée pour tout intervenant quel que soit les domaines où il intervient (sport de compétition, animation sportive, loisir, intervention dans le milieu de handicap...). Une question enfin, qui faute d'avoir été clairement posée jusque là, n'a donc jamais été prise en charge dans le cadre des dispositifs et stratégies de formation mis en place au sein de notre UFR APS et a été, par conséquent, systématiquement renvoyée à la responsabilité personnelle de chaque étudiant.

\section{Description du projet de formation:}

Cette dimension de la formation relève de la formation par projet à l'image de celles actuellement mises en oeuvre dans les formations médicales et dans les écoles d'ingénieurs (Germinet, 2004). Elle vise en effet à rendre les étudiants capables de choisir en fonction des problèmes spécifiques qu'ils vivent à un moment donné, à acquérir les habilités et les connaissances dont ils ont besoin pour assurer les responsabilités afférentes à un rôle social particulier (Legendre, 1993). Des projets dont la thématique est fixée par les formateurs et qui visent l'acquisition de connaissances précises sur les APS et préalablement arrêtées par ces derniers (Bireaud, 1990).

\section{1. Caractéristiques générales}

Les enseignements sont en effet programmés dans le but de leur faire acquérir ce qu'il y a d'essentiel à «savoir » sur l'action humaine lorsque celle-ci est impliquée dans les pratiques physiques de types sportive et artistique et ce pour pouvoir, à terme, élaborer 
des dispositifs d'enseignements pertinents c'est à dire susceptibles de faire progresser significativement des pratiquants qui ne sont pas spécialistes de ces activités.

Ces enseignements font appel à la pratique des APSA et dans ce cadre à l'intervention auprès de pairs. Le recours à la pratique des activités physiques et à l'intervention est cependant à considérer ici essentiellement, mais non exclusivement, en tant que moyen pour faire acquérir des connaissances sur les APSA. En ce sens, ils ne se confondent pas avec d'autres enseignements qui sont programmés dans le but de rendre les étudiants plus performants dans la pratique d'un sport ou de leur apprendre à l'enseigner dans le cadre des stages en milieu scolaire ou associatif. Ils s'adressent enfin à tous les étudiants et occupent une place prépondérante dans la maquette avec plus du tiers des 180 ECTS (European Credit Transfert System) qui la composent.

2. 2. La programmation de ces enseignements.

Les enseignements programmés dans cette dimension de la formation sont structurés en deux niveaux. Le premier niveau couvre les deux premières années de formation, le second la troisième année.

A chacun de ces niveaux le développement d'une compétence particulière est visé en référence à un type de décision à prendre en tant qu'enseignant et qui concerne ce qu'il conviendrait de faire faire à des pratiquants pour essayer de les faire progresser d'une manière significative. Le développement de ces compétences suppose :

- l'implication des étudiants dans des situations où ils ont à analyser la prestation de ces pratiquants en vue de leur proposer des tâches susceptibles de les faire progresser ;

- un changement de mode d'analyse par la mobilisation d'un nouveau corps de connaissances (ou savoir) sur les actions à enseigner; savoirs que nous qualifierons de technologiques en référence au concept de praxéologie ou d'organisation praxéologique emprunté à Chevallard (1995).

Ces compétences, modes d'analyse et connaissances sont présentés par niveau de formation dans les deux tableaux suivants. Ceux-ci sont extraits de la documentation donnée au membre du groupe programme en charge des enseignements relevant de cette dimension de la formation. 
Tableau I. Niveau 1- Licence 1 \& 2

Compétence à développer:

Identifier les modifications techniques ${ }^{2}$ à faire opérer à des pratiquants pour que, en fonction de leur niveau de départ, ils puissent progresser significativement dans la pratique d'une activité sportive ou artistique donnée et définir les «contextes» d'action dans lesquels les placer pour les rendre « nécessaires ».

Changement de mode d'analyse de la prestation des pratiquants

Passer d'une analyse par ...à... une analyse, de type diagnostic, visant comparaison des gestes produits par les pratiquants avec les normes techniques telles que connues de l'étudiant à cerner dans le fonctionnement des actions produites par les pratiquants ce qui les empêche d'accéder à la performance attendue et pourquoi il en est ainsi

Qui suppose de pouvoir:

- se référer aux modélisations du fonctionnement des différents types actions produites par les pratiquants dans les APSA enseignées

- maîtriser les connaissances à partir desquelles ces modélisations ont pu être opérées.

Tableau II. Niveau 2. Licence 3

Compétence à développer:

Identifier l'origine des difficultés récurrentes rencontrées par les pratiquants pour opérer de telles modifications techniques (les obstacles techniques) et définir les « contextes » d'action dans lesquels les solliciter pour les aider à les surmonter

Changement de mode d'analyse de la prestation des pratiquants

Passer de leur imputation à ...à... Leur localisation du côté des certaines caractéristiques des élèves ou au contexte dans lequel se déroule l'enseignement techniques elles mêmes, en envisageant les incidences de leur mise en œuvre sur le fonctionnement de la motricité impliquée dans la production de tout mouvement humain.

Qui suppose de pouvoir :

- se référer aux modélisations de la motricité impliquée dans la production des différents types d'actions sportives ou artistiques

- maîtriser les connaissances à partir desquelles ces modélisations ont pu être opérées.

\section{3. Organisation}

Les enseignements sont structurés à chacun des niveaux en cinq unités d'enseignement (UE), une par groupement d'activités que, par tradition, nous avons continué à intituler sports en milieu stable, sports de pleine nature, sports collectifs, sports duels et

2 La notion de technique est entendu au sens large de moyen pour réaliser une tâche. II englobe par conséquent les notions de tactique et stratégie. 
danse contemporaine. Toutefois, au premier niveau, si les étudiants doivent suivre toutes les UE programmées, au second niveau, ils peuvent en suivre ou deux ou quatre en fonction de la spécialité du diplôme de licence préparée (enseignement, entraînement...

Le temps de travail que les étudiants doivent consacrer à chacune de ces UE est estimé à 120 heures : 60 heures environ sont réservées à des cours, 60 heures à un travail personnel ou de groupes non directement encadré. Les 60 heures de travail encadré sont structurées en un tiers de Travaux Pratiques (TP), de Travaux Dirigés (TD) et de Cours Magistraux (CM) :

- les Travaux Pratiques sont les lieux de confrontation à des situations de type micro enseignement (Altet, 1991) où, dans une Activité Physique de Sportive ou Artistique donnée, les étudiants ont à y occuper alternativement les positions de pratiquant et d'enseignant dans des conditions aménagées.

- les Travaux Dirigés sont des lieux d'analyse de ces situations, du contenu qui y a été programmé et ce en vue de le faire évoluer.

- les Cours Magistraux sont quant à eux des lieux où sont présentés les modèles à mobiliser pour analyser la prestation des pratiquants en rapport avec le thème retenu et les connaissances qui ont permis de les construire.

Ce dispositif s'appuie sur des préparations de séances que les étudiants doivent opérer en dehors de la présence de l'enseignant. Celles-ci peuvent imposer une recherche documentaire en bibliothèque et/ou un retour sur le travail fait en TD ou TP afin de pouvoir l'intégrer dans la préparation de séance.

\section{3 . Caractéristiques de la novation introduite}

Mettre les étudiants dans une position d'intervention dans un cadre aménagé et quelque peu contrôlable par l'institution de formation n'est pas chose nouvelle. La novation réside plutôt dans le fait de programmer un tel enseignement dès l'entrée à l'université, de lui donner une place plus centrale dans la formation en lui accordant plus du tiers du temps total de la formation et de le faire accéder au statut de formation fondamentale en STAPS au même titre que les sciences et la spécialité sportive. Ce changement de place et de statut inscrit cet enseignement en rupture avec trois idées sur lesquelles reposait jusque là l'architecture de nos formations et qui, faute d'avoir été explicitées, n'ont été jusqu'alors que rarement discutées.

La première idée consiste à penser qu'ils y auraient des pré-requis à satisfaire avant de pouvoir mettre les étudiants en position d'enseignement des Activités Physiques Sportives 
ou Artistiques dans le cadre de la formation ; des pré-requis que l'on peut schématiquement résumer à :

- l'appropriation d'un certain nombre de connaissances sur chacune des Activités Physiques et Sportives à l'image de celles qui sont proposées dans l'ouvrage coordonné par Thépaud (2004) ;

- l'accession à une maîtrise pratique personnelle qui soit suffisante, et dans un spectre assez large d'activités, de techniques corporelles allant bien au delà de celles qu'ils auront à enseigner dans les institutions où ils seront ultérieurement recrutés ;

- avoir « vécu » en tant que pratiquant des situations d'apprentissage de ces techniques dans le cadre d'enseignements conçus et animés par les formateurs de I'UFRAPS.

Le projet de formation global que nous mettons en place aujourd'hui prend en compte cette exigence théorique et pratique mais d'une manière concourante à la formation technologique et, pour ce qui est des connaissances réglementaires et techniques, en sollicitant leur étude d'une manière plus autonome dans le cadre du travail personnel demandé aux étudiants. Les temps d'enseignement étant plutôt réservés à leur manipulation dans le cadre de l'analyse des prestations des élèves ou de la conception des dispositifs d'enseignement .

La seconde idée est relative à la nature des connaissances sur les APSA dont devrait disposer un intervenant non spécialiste de ces activités (polyvalent). Elle consiste à penser que celles-ci se limiteraient à l'appropriation d'informations de natures différentes mais succinctes sur le plus grand nombre d'activités physiques possibles parmi celles que les étudiants auraient ultérieurement le plus probablement à enseigner. Nous pensons au contraire ici que, pour pouvoir les amener à comprendre les différents dispositifs d'intervention à mettre en place en vue de favoriser l'apprentissage des différentes techniques sportives et à les contrôler au plan épistémologique, ce sont donc des modèles abstraits que ces intervenants doivent s'approprier.

Le troisième idée consiste à penser qu'il serait possible de faire accéder les étudiants à la maîtrise de connaissances (modèles) sur les activités physiques indépendamment des problématiques qui ont pu les faire naître, qui leur donnent leur sens et qui touchent ici au fait d'avoir à les enseigner à des publics de non spécialistes. Des informations étaient certes déjà apportées aux étudiants sur la motricité impliquée dans la réalisation des gestes ainsi que sur ce qui permettait de comprendre la portée des techniques sportives ou artistiques, mais lors d'enseignements où ils étaient eux mêmes sollicités en tant que pratiquant dans le but d'optimiser leur performance par la maîtrise pratique de techniques 
corporelles. Coupées des situations pratiques par rapport auxquelles ces connaissances ont été socialement établies pour y agir plus efficacement, cet apport informatif était plus à mémoriser en tant que tel qu'à devoir être utilisé.

En plaçant les étudiants dans une position où ils ont à intervenir auprès de leurs pairs, les enseignements dispensés dans le cadre de la dimension technologique de la formation proposée en premier cycle dans le domaine des Sciences et Techniques des Activités Physiques et Sportives, entendent les inciter à utiliser à bon escient les connaissances produites sur l'action humaine afin de pouvoir leur proposer des dispositifs d'enseignement susceptibles de les faire progresser significativement. La prise en charge de cette forme d'appropriation de connaissances portant sur les actions que produisent les humains dans ce secteur d'activité répond à la volonté d'aider les étudiants à surmonter une des difficultés récurrentes constatée aussi bien chez les étudiants à l'issue de la licence que chez les professionnels en exercice. Cela consiste à ne pas savoir mobiliser les connaissances sur les Activités Physiques Sportives, Artistiques ou d'Entretien dès lors que celles-ci se démarquent des aspects gestuels les plus immédiatement perceptibles.

De multiples difficultés et réticences font en effet obstacle à la mise en place de ce projet de formation. Si les réticences semblent être inhérentes au procès de travail que nous avons engagé, à sa nature même mais aussi aux maladresses que nous avons commises au niveau de son pilotage. Néanmoins, les difficultés ont quant à elles pour principale origine le fait que les modèles et les connaissances dont il est question ici n'existent aujourd'hui que d'une manière lacunaire et éparses dans les productions écrites ou pratiques de certains experts ou leaders d'opinion (Robin, 2003). Ceux-ci, plus particulièrement impliqués en position de formateur dans des institutions, visent la démocratisation de l'enseignement de leur sport ou art. L'objectivation et l'institutionnalisation de ces connaissances en un corps (savoirs) dûment établi relèvent donc de la responsabilité des formateurs qui s'y engagent et, au delà, de ces institutions universitaires que sont les UFR APS.

Surmonter cette difficulté constitue un réel défi. Notre capacité à le relever est encore incertaine, elle suppose en plus de ce travail d'ingénierie, que l'on se retourne du côté des personnes et des institutions. En particulier, celles qui, depuis plusieurs générations ont consacré leur vie (plus que leur vie professionnelle) à la diffusion de leur sport favori par le truchement de son enseignement et à prendre au sérieux les connaissances (sur ces activités) qu'ils ont dû construire le plus souvent par un savant bricolage afin de «fabriquer» des procédés d'intervention toujours plus efficaces. Elle suppose également 
la poursuite de recherches dans la lignée de celles initiées par exemple par Robin (1998) ${ }^{3}$ et Vandevelde (2003) sur les leaders d'opinion et de celles sur les savoirs professionnels proposées par Dugal et Leziart (2005). Elle suppose enfin de s'ouvrir à d'autres problématiques comme celles concernant l'utilisation des sciences humaines dressées par Lessard et al (2004).

Relever un tel défi est nécessaire si l'on veut qu'à moyen terme, les formations universitaires en STAPS, puissent, à leur manière, avoir une réelle et irremplaçable utilité sociale au regard de la formation des futurs professionnels dans le domaine des métiers du sport et de l'Education Physique.

\section{Bibliographie.}

Altet, M., \& Britten, D. (1991). Micro- enseignement et formation des enseignants. Paris : PUF.

Ardouin, T. (2003). Ingénierie de formation pour l'entreprise. Paris : Dunod.

Amade-Escot, C. (1998). Apport des recherches en didactique à l'analyse de l'enseignement de l'EPS. Une étude de cas : le contrat. In C. Amade-Escot \& al, Recherches en éducation physique et sportive : bilan et perspectives (pp 253266). Paris : Revue EPS.

Amade-Escot, C. (2001). De l'usage des théories de l'enseignant. Questions posées par l'étude des contrats didactiques en éducation physique. In A.Mercier, \& al, Le génie didactique, usages et mésusages des théories de l'enseignement (pp 2341). Bruxelles : De Boeck.

Amade-Escot, C. (2003). La gestion interactive du contrat didactique en volley-ball: agencement des milieux et régulations du professeur. In C.Amade-Escot, C, Didactique de l'éducation physique, état des recherches (pp 255-278).Paris: Revue EPS.

Amade-Escot, C., \& Léziart, Y. (1996). Contribution à l'étude de la diffusion de propositions d'ingénierie didactique auprès de praticiens. Analyse de cas d'enseignants d'éducation physique et sportive volontaires. Rapport scientifique. Recherche INRP, n³0506.Mai.

Amade-Escot, C., \& Marsenach, J. (1995). Didactique de l'éducation physique et sportive : questions théoriques et méthodologiques. Grenoble : LA Pensée Sauvage.

${ }^{3}$ Auquel nous devons notre premier contact avec la notion d'îlot de rationalité. 
Attiklémé, K. (2002). Programme d'enseignement de la natation dans les collèges: contraintes et conditions d'élaboration. Le point de vue de l'anthropologie didactique. Thèse de doctorat en STAPS, non publiée, université Joseph Fourier, Grenoble 1.

Bireaud, A. (1990). Les méthodes pédagogiques dans l'enseignement supérieur. Paris: Les Organisations.

Camy, J. (2005). Le dispositif LMD dans son contexte européen. Revue EPS, 313, 8-10. Chevallard, Y. ( 1995). La fonction professorale : esquisse d'un modèle didactique. In Ecole et université d'été de didactique des mathématiques (pp 83-122). Actes de l'école d'été, Saint- Sauves d'Auvergne.

Collinet,C. (2005). Quels savoirs scientifiques les enseignants d'EPS et les entraîneurs jugent-ils utiles ? Revue Française de Pédagogie, 150,43-58

Dugal, J.P. \& Léziart, Y. (2004). La circulation des savoirs entre recherche et formation : l'exemple des concepts didactiques lors d'une action de formation de conseillers pédagogiques. Revue Française de Pédagogie, 149, 37-47.

Durand, M. (1996). Recherches en enseignement et formation des professeurs d'éducation physique et sportive. In Recherche et pratique des APS. Dossiers EPS, 28, 78-84.

Durand, M. (2001). Chronomètre et survêtement. Reflets de l'expérience quotidienne d'enseignants en Education Physique. Paris : Revue EPS.

Germinet, R. (2004). L'ingénieur au chevet de la démocratie. Paris : O. Jacob.

Jonnaert, P. (2003). Perspectives curriculaires contemporaines et changements des rapports aux savoirs. In S.Maury,\& M.Caillot (Eds), Rapport au savoir et didactiques (pp 105-122). Paris : Fabert.

Le Boterf, G. (2004). L'ingénierie : concevoir des dispositifs dans des environnements complexes et évolutifs. In Où en est l'ingénierie de formation ? Education Permanente, 157, 53-62.

Legendre, R. ( 1993). Dictionnaire actuel de l'éducation. 2ème édition. Paris / Montréal : Guerin

Lessard, C., Altet, M., Paquay, L,., Perrenoud, P. (2004). Entre sens commun et sciences humaines. Quels savoirs pour enseigner ? Bruxelles : De Boeck.

Mercier, A., Lemoyne, G., Rouchier, A. (2001). Le génie didactique. Usages et mésusages des théories de l'enseignement. Bruxelles : De Boeck. 
Robin, J-F. (2003). Transposition didactique : le rôle des leaders en gymnastique scolaire. In C. Amade-Escot (Ed.) Didactique de l'éducation physique, état des recherches (pp 27-48). Paris : Revue EPS

Siedentop,D. (1989-2002). Content Knowledge for Physical Education. Journal of teaching in physical education, 21,368-377.

Terral, P. (2005) : Le LMD. La réforme universitaire en sciences du sport. Revue EPS, 313, 5-6.

Thépaud, A.,\& al (2004). Les APS en licence STAPS: théorie des pratiques d'apprentissage. Paris : Masson.

Vandevelde, M. (2003). Des pratiques sociales du basket-ball à un savoir d'expert: construction d'une épistémologie scolaire renouvelée. In C. Amade-Escot (Ed.) Didactique de l'éducation physique, état des recherches (pp 49-78). Paris: Revue EPS. 
Annexe

Enseignements programmés dans les différentes dimensions du tronc commun de la formation

\begin{tabular}{|c|c|c|c|}
\hline & Dimension scientifique & Dimension technologique & Dimension sportive \\
\hline \multirow[t]{4}{*}{$\mathrm{L} 1$} & Physiologie de l'effort sportif & $\begin{array}{l}\text { Technologie enseignement des sports en } \\
\text { milieu stable Niveau } 1\end{array}$ & Spécialité sportive niveau 1 \\
\hline & Mécanique des gestes sportifs & $\begin{array}{l}\text { Technologie enseignement des sports collectifs } \\
\text { Niveau } 1\end{array}$ & \\
\hline & Sciences cognitives et APS & $\begin{array}{l}\text { Technologie enseignement de la condition } \\
\text { physique aspects organiques }\end{array}$ & \\
\hline & & $\begin{array}{l}\text { Technologie enseignement de la condition } \\
\text { physique aspects musculaires }\end{array}$ & \\
\hline \multirow[t]{4}{*}{$\mathrm{L} 2$} & $\begin{array}{l}\text { Dimension sociale des pratiques } \\
\text { corporelles }\end{array}$ & $\begin{array}{l}\text { Technologie enseignement des Activités } \\
\text { Physiques de Pleine nature Niveau } 1\end{array}$ & Dimension sportive niveau 2 \\
\hline & Approche pluridisciplinaire de la motricité & $\begin{array}{l}\text { Technologie enseignement des activités } \\
\text { duelles Niveau } 1\end{array}$ & \\
\hline & $\begin{array}{l}\text { Approche pluridisciplinaire du } \\
\text { développement }\end{array}$ & $\begin{array}{l}\text { Technologie enseignement de la condition } \\
\text { physique aspects psychotoniques }\end{array}$ & \\
\hline & & $\begin{array}{l}\text { Technologie enseignement de la danse } \\
\text { contemporaine Niveau } 1\end{array}$ & \\
\hline \multirow[t]{2}{*}{ L3 } & $\begin{array}{l}\text { Approche pluridisciplinaire des facteurs } \\
\text { de la performance motrice }\end{array}$ & $\begin{array}{l}\text { Technologie enseignement niveau } 2 \\
\text { APS } 1 *\end{array}$ & Spécialité sportive niveau 3 \\
\hline & $\begin{array}{l}\text { Approche pluridisciplinaire des } \\
\text { apprentissages moteurs }\end{array}$ & $\begin{array}{l}\text { Technologie enseignement niveau } 2 \\
\text { APS } 2 *\end{array}$ & \\
\hline
\end{tabular}

* A choisir parmi : APPN/ Activités Duelles/ Danse contemporaine / Sports collectifs / Sports en milieu stable 